\title{
Upper Esophageal Sphincter Compression Device as an Adjunct to Proton Pump Inhibition for Laryngopharyngeal Reflux
}

\author{
Rena Yadlapati ${ }^{1}\left[\right.$. John E. Pandolfino ${ }^{2} \cdot$ Madeline Greytak $^{1} \cdot$ Jonathon Cahoon $^{3} \cdot$ Mary Clarke $^{1} \cdot$ Matthew Clary $^{4}$. \\ Daniel Fink ${ }^{4} \cdot$ Paul Menard-Katcher ${ }^{3} \cdot$ Andrew M. Vahabzadeh-Hagh $^{5} \cdot$ Philip Weissbrod $^{5}$. Samir Gupta ${ }^{1}$. \\ Alexander Kaizer ${ }^{6} \cdot$ Sachin Wani ${ }^{3}$
}

Received: 13 May 2021 / Accepted: 8 July 2021 / Published online: 18 July 2021

(c) The Author(s), under exclusive licence to Springer Science+Business Media, LLC, part of Springer Nature 2021

\begin{abstract}
Background The Reflux Band, an external upper esophageal sphincter (UES) compression device, reduces esophago-pharyngeal reflux events. This study aimed to assess device efficacy as an adjunct to proton pump inhibitor (PPI) therapy in patients with laryngopharyngeal reflux (LPR).

Methods This two-phase prospective clinical trial enrolled adults with at least 8 weeks of laryngeal symptoms (sore throat, throat clearing, dysphonia) not using PPI therapy at two tertiary care centers over 26 months. Participants used double dose PPI for 4 weeks in Phase 1 and the external UES compression device nightly along with PPI for 4 weeks in Phase 2. Questionnaire scores and salivary pepsin concentration were measured throughout the study. The primary endpoint of symptom response was defined as reflux symptom index (RSI) score $\leq 13$ and/or $>50 \%$ reduction in RSI.

Results Thirty-one participants completed the study: 52\% male, mean age 47.9 years (SD 14.0), and mean body mass index (BMI) $26.2 \mathrm{~kg} / \mathrm{m}^{2}(5.1)$. Primary endpoint was met in 11 (35\%) participants after Phase 1 (PPI alone) and 17 (55\%) after Phase 2 (Device + PPI). Compared to baseline, mean RSI score (24.1 (10.9)) decreased at end of Phase 1 (PPI alone) (21.9 (9.7); $p=0.06)$ and significantly decreased at end of Phase 2 (Device + PPI) (15.5 (10.3); $p<0.01)$. Compared to non-responders, responders to Device + PPI had a significantly lower BMI $(p=0.02)$ and higher salivary pepsin concentration $(p=0.01)$. Conclusion This clinical trial highlights the potential efficacy of the external UES compression device (Reflux Band) as an adjunct to PPI for patients with LPR (ClinicalTrials.Gov NCT03619811).
\end{abstract}

Keywords Gastroesophageal reflux disease $\cdot$ Extra-esophageal reflux $\cdot$ Peptest $\cdot$ Ambulatory reflux monitoring

Rena Yadlapati

ryadlapati@ucsd.edu

1 Division of Gastroenterology, University of California, San Diego School of Medicine, ACTRI Building 1W517, 9500 Gilman Drive MC 0956, La Jolla, CA 92093, USA

2 Division of Gastroenterology, Northwestern University, Feinberg School of Medicine, Chicago, IL, USA

3 Division of Gastroenterology, University of Colorado, Anschutz Medical Campus, Aurora, CO, USA

4 Division of Otolaryngology, Department of Surgery, University of Colorado, Anschutz Medical Campus, Aurora, $\mathrm{CO}$, USA

5 Division of Otolaryngology, Department of Surgery, University of California San Diego, La Jolla, CA, USA

6 Department of Biostatistics and Informatics, Colorado School of Public Health, University of Colorado, Anschutz Medical Campus, Aurora, CO, USA

$\begin{array}{ll}\text { Abbreviations } \\ \text { BMI } & \text { Body mass index } \\ \text { EGJ } & \text { Esophagogastric junction } \\ \text { GERD } & \text { Gastroesophageal reflux disease } \\ \text { GerdQ } & \text { GERD questionnaire } \\ \text { IRP } & \text { Integrated relaxation pressure } \\ \text { LFD } & \text { Lateral flow device } \\ \text { LPR } & \text { Laryngopharyngeal reflux } \\ \text { PPI } & \text { Proton pump inhibitor } \\ \text { RSI } & \text { Reflux symptom index } \\ \text { SD } & \text { Standard deviation } \\ \text { UES } & \text { Upper esophageal sphincter } \\ \text { Reflux Band } & \text { UES compression device }\end{array}$




\section{Introduction}

One-third of the adult US population experiences laryngeal complaints such as throat clearing, dysphonia, or chronic cough. A vast majority of these individuals (up to $80 \%$ ) who undergo workup will receive a diagnosis of laryngopharyngeal reflux (LPR) [1-4]. LPR is considered an extra-esophageal syndrome of GERD in which retrograde reflux of gastric contents proximal to the upper esophageal sphincter (UES) leads to chronic laryngeal irritation [5-7]. The assessment and management of suspected LPR are challenging with patients visiting an average of ten specialists and undergoing six tests in the initial year of evaluation, often without diagnostic clarity or improvement [8-12]. The current paradigm for LPR contributes to significant health care costs, estimated at over 50 billion US dollars annually [11].

A major challenge for clinicians and patients relates to the variable and suboptimal efficacy of conventional, often empiric, medical, and surgical anti-reflux therapies for LPR [13, 14]. The majority of patients with suspected LPR are trialed on proton pump inhibitor (PPI) therapy, though fewer than $50 \%$ will derive symptom relief [15, 16]. Some patients not responsive to PPI therapy are referred for invasive procedures such as surgical fundoplication, although outcomes following anti-reflux surgery in patients with LPR are poor [17-19]. Despite effectively suppressing gastric acid secretion, PPI therapy does not reduce the burden of gastroesophageal refluxate. Similar to concepts in gastroesophageal reflux disease (GERD), gastric refluxate is composed of bile and pepsin, which can injure esophageal and laryngeal epithelium, independent of acidity [20]. Thus, safe and less invasive treatment options beyond acid suppression are needed for LPR.

Incompetence of the UES to restrict passage of esophageal refluxate is considered an important mechanism in the development of LPR [21, 22]. In 2014, Shaker and colleagues demonstrated that external application of a device at the cricoid augmented UES pressure and reduced esophago-pharyngeal reflux events [23]. These concepts led to the development of the Reflux Band (Somna Therapeutics, Germantown, WI; previously referred to as the Reza Band), an FDA cleared external UES compression device worn around the neck at night which applies cricoid pressure to generate $20-30 \mathrm{mmHg}$ intraluminal UES pressure and augment UES competence [24]. While the external UES compression device is a seemingly safe and noninvasive therapy for LPR, clinical adoption has been slow due to paucity of efficacy data and uncertainty surrounding clinical role. We hypothesize that the unique complimentary mechanisms of the external UES compression device in conjunction with acid suppression is an effective novel therapeutic strategy for patients with LPR. Thus, in this two-phase clinical trial, we aimed to assess efficacy of the external UES compression device as an adjunct to PPI therapy in patients with LPR.

\section{Methods}

\section{Study Design}

This two-phase prospective proof-of-concept clinical trial enrolled participants over 26 months (August 2018 to October 2020) at two tertiary care centers (University of California San Diego (UCSD), La Jolla, CA; University of Colorado, Aurora, CO). The study was approved by the institutional review board at each site and registered with Clinical Trials Gov NCT03619811.

\section{Participants}

English and Spanish speaking male and female adults (18-89 years) experiencing 8 weeks or more of laryngeal symptoms (throat clearing, sore throat, dysphonia, cough) not on PPI therapy were enrolled. If on PPI therapy at time of enrollment, participants went through a washout of at least 8 weeks off PPI prior to study start. Exclusion criteria included history of prior foregut surgery, a diagnosis of achalasia, laryngeal mass lesion on prior laryngoscopy, pregnancy, breastfeeding, intolerance to PPI, imprisonment, or inability to consent. Patients who met a contraindication to the external UES compression device per manufacturer guidelines were also excluded: implants or implant parts that reside in the area where device is applied, pacemaker, cardiovascular defibrillator, vagus nerve stimulator; a diagnosis of glaucoma; malignancy of neck, including neck surgery; altered mental status; carotid artery disease; thyroid disease; history of cerebrovascular disease; connective tissue disorder (i.e., Marfan's Syndrome or Ehlers-Danlos Syndrome); current use of nocturnal noninvasive ventilation machines (e.g., continuous positive airway pressure).

\section{Study Protocol (Fig. 1)}

Eligible patients seen at the gastroenterology and/or laryngology clinics that provided informed consent were enrolled. At enrollment participants completed the reflux symptom index (RSI) and GerdQ instruments, answered questions about their current symptoms, provided a $2 \mathrm{~mL}$ fasting saliva sample while on PPI for pepsin analysis, and started Phase 1 of the study. During Phase 1 (PPI alone), participants took PPI therapy (equivalent of Omeprazole $40 \mathrm{mg}$ daily) for 4 weeks, completing two phone calls with the research coordinator at weeks 1 and 3 . At 


\begin{tabular}{|c|c|c|c|c|}
\hline $\begin{array}{l}\text { Enrollment } \\
\text { Week } 0\end{array}$ & $\begin{array}{c}\text { Phase } 1 \text { (PPI alone) Weeks } \\
\text { o to } 4\end{array}$ & $\begin{array}{l}\text { Mid-Study Visit } \\
\text { Week } 4\end{array}$ & $\begin{array}{l}\text { Phase } 2 \text { (Device+PPI) } \\
\text { Weeks } 4 \text { to } 8\end{array}$ & $\begin{array}{c}\text { End of Study Visit } \\
\text { Week } 8\end{array}$ \\
\hline $\begin{array}{l}\text { - Questionnaires } \\
\text { - Saliva collection } \\
\text { - If esophageal } \\
\text { physiologic testing } \\
\text { performed as standard } \\
\text { of care within } 3 \text { months, } \\
\text { data collected }\end{array}$ & $\begin{array}{l}\text { Double dose PPI daily } \\
\text { - } 2 \text { research coordinator } \\
\text { calls (weeks } 1 \text { and } 3 \text { ) }\end{array}$ & $\begin{array}{l}\text { - External UES } \\
\text { compression device } \\
\text { fitting \& education } \\
\text { - Questionnaires }\end{array}$ & $\begin{array}{l}\text { - Nightly use of Device }+ \\
\text { Daily use of double } \\
\text { dose PPI } \\
\text { - } 2 \text { research coordinator } \\
\text { calls (weeks } 5 \text { and } 7 \text { ) }\end{array}$ & $\begin{array}{l}\text { - Follow-up Clinic Visit } \\
\text { - Questionnaires }\end{array}$ \\
\hline
\end{tabular}

Fig. 1 Study protocol. This two-phase 8-week study protocol included baseline data collection at enrollment, 4 weeks of PPI use alone in Phase 1, a mid-study visit at week 4 for data collection and

the end of Phase 1, participants returned for external UES compression device education and fitting per manufacturer guidelines and completed the RSI and GerdQ. Next participants entered into Phase 2 (Device + PPI). During Phase 2 participants used the external UES compression device nightly and continued PPI therapy for 4 weeks, completing two phone calls with the research coordinator at weeks 5 and 7. Research coordinator calls during the study were to assess safety and protocol adherence. At the end of Phase 2 participants returned for a follow-up clinic visit, and completed the RSI, GerdQ, and feedback questionnaire. Follow-up calls, optional for participants, were performed at 3 months following completion of Phase 2 to assess continued use of PPI and/or external UES compression device.

\section{Data Collection}

Data for all subjects were collected in de-identified datasets on institutional Research Electronic Data Capture databases at both sites. Data collection included demographics, clinical data, and results of esophageal physiologic testing if available. While not required by this protocol, high-resolution esophageal manometry and/or ambulatory reflux monitoring off PPI is routinely performed in this patient population and such data were abstracted if available within a 3-month period of study enrollment.

\section{Questionnaires}

Participants completed two validated instruments, the RSI and GerdQ, at baseline, following Phase 1 and following Phase 2 . The nine-item RSI is an externally validated patient-reported instrument to evaluate LPR symptom burden, with higher scores on the range from 0 to 45 indicating more severe symptoms. An RSI score greater than 13 is indicative of elevated LPR symptom burden [25]. The sixitem GerdQ evaluates reflux symptoms over a 7-day period, with higher scores on the range from 0 to 18 indicating more severe symptoms [26]. external UES compression device fitting, 4 weeks of external UES compression device use with PPI in Phase 2, and an end of study visit at week 8 for data collection and follow-up

\section{Salivary Pepsin}

Unstimulated expectorated saliva samples were collected from subjects to measure salivary pepsin, a noninvasive biomarker of GERD, per our previously described protocol [27]. Saliva samples were placed into $15-\mathrm{mL}$ sterile plastic tubes containing $0.5 \mathrm{~mL}$ of $0.01 \mathrm{~mol} / \mathrm{L}$ citric acid at $\mathrm{pH} 2.5$. The samples were promptly transferred to the refrigerator at $4{ }^{\circ} \mathrm{C}$. Pepsin was measured using the Peptest lateral flow device (LFD) (RD Biomed Ltd). Within 7 days of collection, samples were centrifuged for $5 \mathrm{~min}$ at 4,000 rpm in a bench top centrifuge, and the supernatants were collected. $80 \mu \mathrm{L}$ of the supernatants layer was then mixed with $240 \mu \mathrm{L}$ of migration buffer solution and vortexed for $10 \mathrm{~s}$. Then, $80 \mu \mathrm{L}$ of the mixture was added to the well of the LFD. The LFD was transferred to the Peptest recorder which provided a quantified concentration of pepsin in $\mathrm{ng} / \mathrm{ml}$. Peptest has the ability to detect pepsin concentrations of $16 \mathrm{ng} / \mathrm{mL}$ or greater. Concentrations between 16 and $24.9 \mathrm{ng} / \mathrm{mL}$ are quantified as $16<25 \mathrm{ng} /$ $\mathrm{mL}$ by the recorder.

\section{External UES Compression Device Fitting}

The external UES compression device is a novel therapy developed for GERD and LPR. It is composed of a plastic frame (front facing), a comfort band made of soft and pliable material which is adjustable by Velcro on each side. There is a magnetic clasp for easily securing and removing the device without readjusting the pressure. A soft foam cushion applies pressure to the cricoid cartilage. Small increments of pressure can be adjusted with a front facing dial. The device is fitted with an external manometer and pressure sensor, in which the blue pressure sensor is gently placed underneath the cushion of the device. In this study, the device was fitted to generate a pressure of $20-30 \mathrm{mmHg}$ as per manufacturer guidelines. 


\section{Outcomes and Statistical Analysis}

The primary outcome was LPR symptom response, measured by the RSI score, categorized as response or nonresponse. Symptom response was defined as an RSI of 13 or less and/or a 50\% reduction from baseline RSI.

The primary analysis included participants that completed the entire study to assess symptom response (Primary outcome; RSI score). Additionally, association between baseline characteristics and study response was measured to identify factors associated with symptom response. Secondary analysis of all participants that completed Phase 1 , including those that did not complete Phase 2, was performed to analyze the association between baseline characteristics and response in Phase 1, PPI therapy alone. Continuous and categorical summaries are presented as mean (standard deviation) and frequency (percent), respectively. Comparisons between responders and non-responders used simple logistic regression for categorical measures and two sample $t$ tests for continuous measures. All figures and analyses were conducted using R v3.6.3 (Vienna, Austria). All authors had access to the study data and reviewed and approved the final manuscript.

\section{Results}

\section{Participant Baseline Characteristics and Study Flow}

Figure 2 depicts the study flow. In total 43 participants enrolled, 36 completed Phase 1 and 31 completed Phase 2. Primary analysis includes the 31 participants that completed both study phases (Table 1 ). The mean age was 47.9 years (SD 14.0), 16 (52\%) were male, and the mean body mass index (BMI) was $26.2 \mathrm{~kg} / \mathrm{m}^{2}$ (5.1). Participants reported the following laryngeal symptoms at baseline: throat clearing (24,77\%), cough (24,77\%), and voice hoarseness $(21,68 \%)$. With regard to esophageal symptoms, the majority reported regurgitation $(23,74 \%)$ and heartburn $(16,52 \%)$. Nine (29\%) had a hiatal hernia. At enrollment, mean RSI score was 24.1 (SD 10.9), mean GerdQ score was 8.5 (SD 3.4) and salivary pepsin concentration was 58.4 (SD 72.8) $\mathrm{ng} / \mathrm{ml}$.

Ambulatory reflux monitoring off PPI therapy was available for 30/31 (97\%) participants. Mean total acid exposure time (\% time spent below pH of 4.0 ) was $3.2 \%$ (SD 2.5) and mean total number of reflux events was 75.2 (SD 70.7). Seven participants underwent ambulatory reflux monitoring using a dual $\mathrm{pH}$-impedance catheter with a

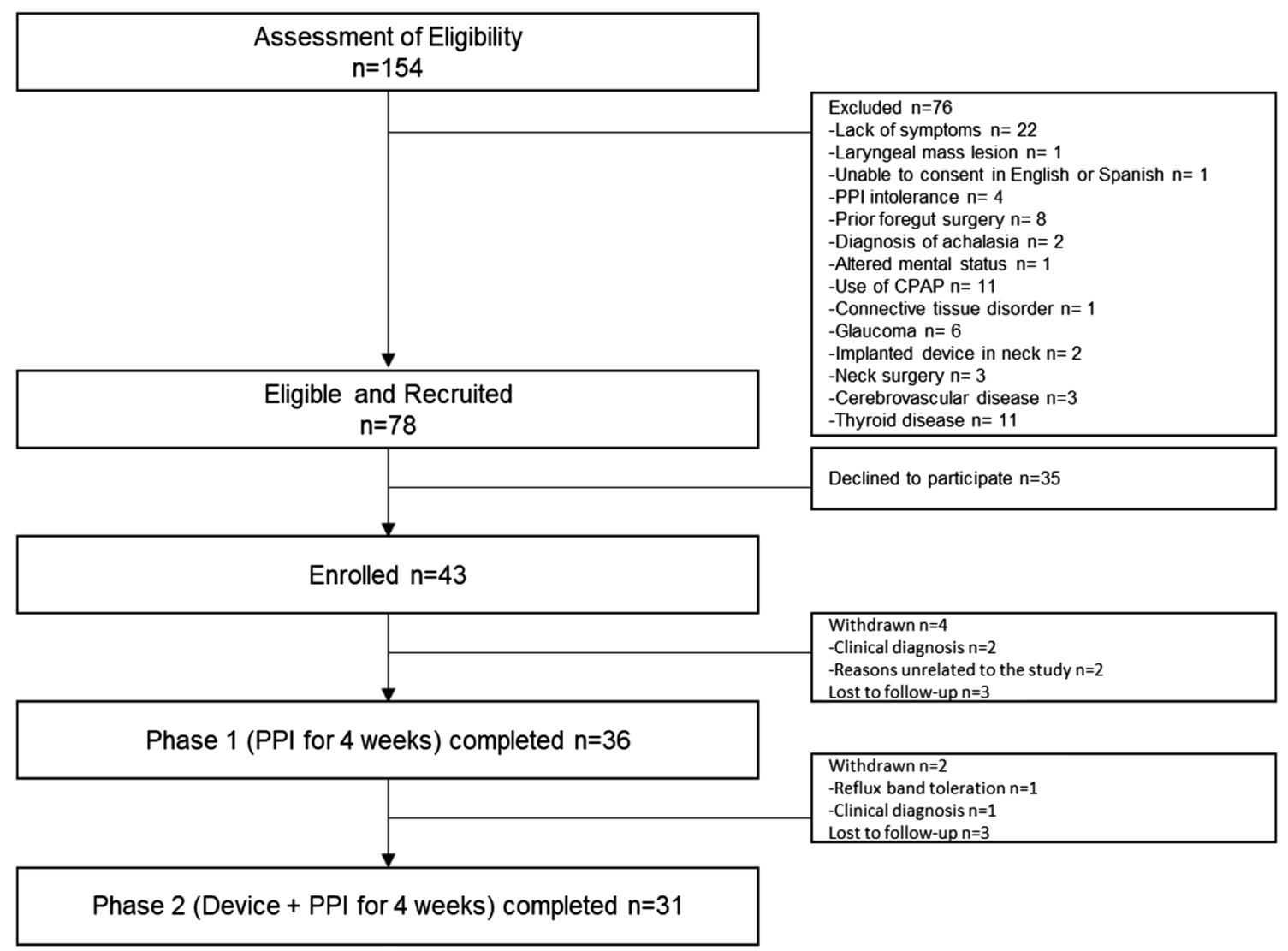

Fig. 2 Participant study flow. Of 43 participants enrolled, 36 completed Phase 1 and 31 completed both Phase 1 and Phase 2 
Table 1 Baseline characteristics

\begin{tabular}{|c|c|}
\hline Variable & $N=31$ \\
\hline Male & $16(51.6 \%)$ \\
\hline Age (years) & $47.9(14.0)$ \\
\hline Body mass index $\left(\mathrm{kg} / \mathrm{m}^{2}\right)$ & $26.2(5.1)$ \\
\hline \multicolumn{2}{|l|}{ Symptoms } \\
\hline Cough & $24(77.4 \%)$ \\
\hline Throat clearing & $24(77.4 \%)$ \\
\hline Voice hoarseness & $21(67.7 \%)$ \\
\hline Globus & $18(58.1 \%)$ \\
\hline Regurgitation & $23(74.2 \%)$ \\
\hline Heartburn & $16(51.6 \%)$ \\
\hline Chest pain & $4 / 21(19.0 \%)$ \\
\hline Dysphagia & $12(38.7 \%)$ \\
\hline Reflux symptom index (RSI) score & $24.1(10.9)$ \\
\hline GerdQ score & $8.5(3.4)$ \\
\hline Salivary pepsin concentration $(\mathrm{ng} / \mathrm{ml})$ & $94.6(116.8)$ \\
\hline \multicolumn{2}{|c|}{ Ambulatory reflux monitoring off PPI $(n=30)$} \\
\hline Total acid exposure time $(\%)$ & $3.2(2.5)$ \\
\hline Acid exposure time $>4.0 \%$ & $11(35.5 \%)$ \\
\hline Total number of reflux events & $75.2(70.7)$ \\
\hline Symptom index & $37.2(33.6)$ \\
\hline Symptom association probability & $91.8(19.0)$ \\
\hline \multicolumn{2}{|l|}{ LPR reflux event monitoring $(n=7)$} \\
\hline Total LPR events & $5.4(5.3)$ \\
\hline Acidic to acidic events & $2.6(3.7)$ \\
\hline Acidic to non-acidic events & $1.9(1.8)$ \\
\hline \multicolumn{2}{|c|}{ High-resolution impedance manometry $(n=25)$} \\
\hline EGJ baseline pressure $(\mathrm{mmHg})$ & $34.2(24.2)$ \\
\hline Hiatal hernia $(>1 \mathrm{~cm})$ & $9(29.0 \%)$ \\
\hline CD-LES separation $(\mathrm{cm})$ & $0.6(0.9)$ \\
\hline Median LES IRP (mmHg) & $13.9(6.7)$ \\
\hline Percent of failed swallows & $5.2(10.5)$ \\
\hline$\%$ of bolus clearance complete & $78.3(31.4)$ \\
\hline UES resting pressure $(\mathrm{mmHg})$ & $18.8(24.9)$ \\
\hline UES residual pressure (mmHg) & $3.5(10.9)$ \\
\hline UES length $(\mathrm{cm})$ & $3.1(0.8)$ \\
\hline
\end{tabular}

Continuous data presented as mean (SD) and categorical data as $n$ (\%)

$P P I$ proton pump inhibitor, $L P R$ laryngopharyngeal reflux, EGJ esophago-gastric junction, LES lower esophageal sphincter, IRP integrated relaxation pressure, UES upper esophageal sphincter

mean number of 5.4 (5.3) LPR events with the following mean breakdown: 2.6 (3.7) acidic to acidic reflux events and 1.9 (SD 1.8) acidic to non-acidic reflux events. Highresolution manometry was available for $25 / 31$ (81\%) participants with a mean UES resting pressure of $18.8 \mathrm{mmHg}$ (SD 24.9), mean baseline EGJ pressure of $34.2 \mathrm{mmHg}$ (SD 24.2), and average median integrated relaxation pressure of $13.9 \mathrm{mmHg}$ (SD 6.7).

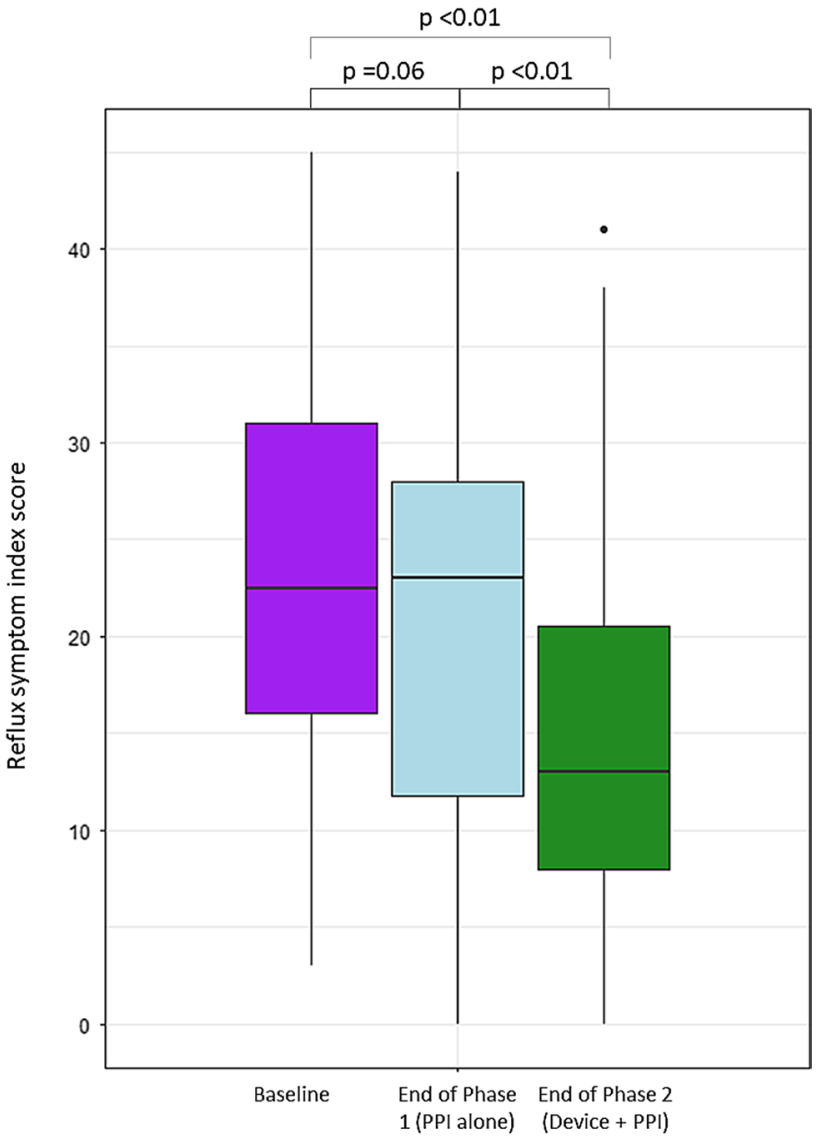

Fig. 3 Box plot of reflux symptom index (RSI) scores throughout the study. Compared to baseline, mean RSI scores did not significantly change during Phase 1 (with PPI alone). Compared to baseline and end of Phase 1, mean RSI scores significantly reduced during Phase 2 (with Device and PPI use)

\section{Primary Analysis}

\section{Symptom Response to UES Compression Device with PPI Therapy}

Compared to RSI score at baseline (24.1 (SD 10.9)), the RSI score decreased following Phase 1 (PPI alone) (21.9 (SD 9.7); $p=0.06)$ and significantly decreased following Phase 2 (Device + PPI) (Phase 2: 15.5 (SD 10.3); $p<0.01$ ). RSI score was also significantly reduced following Phase 2 compared to end of Phase $1(p<0.01)$ (Fig. 3). The mean reduction in RSI during Phase 2 was significantly greater than during Phase 1 (-6.4 (6.3) vs -2.3 (6.4); $p=0.04)$. Symptom response was met in $11 / 31(35 \%)$ at end of Phase 1 (PPI alone) and 17/31 (55\%) at end of Phase 2 (Device + PPI).

\section{Factors Associated with Symptom Response to Device + PPI}

Baseline characteristics between the 17 responders to Device + PPI were compared to the 14 non-responders. 
Table 2 Univariate comparison of baseline variables between responder and non-responders to external UES compression Device + PPI intervention

\begin{tabular}{|c|c|c|c|}
\hline Variable & $\begin{array}{l}\text { Device }+ \text { PPI } \\
\text { non-responder } \\
(N=14)\end{array}$ & $\begin{array}{l}\text { Device }+ \text { PPI responder } \\
(N=17)\end{array}$ & $p$ value \\
\hline Male & $6(42.9 \%)$ & $10(58.8 \%)$ & 0.38 \\
\hline Age (years) & $49.6(12.0)$ & $46.5(15.7)$ & 0.53 \\
\hline Body mass index $\left(\mathrm{kg} / \mathrm{m}^{\mathrm{g}}\right)$ & $28.6(5.3)$ & $24.2(4.1)$ & 0.02 \\
\hline \multicolumn{4}{|l|}{ Baseline symptoms } \\
\hline Cough & $12(85.7 \%)$ & $12(70.6 \%)$ & 0.32 \\
\hline Throat clearing & $12(85.7 \%)$ & $12(70.6 \%)$ & 0.32 \\
\hline Voice hoarseness & $12(85.7 \%)$ & $9(52.9 \%)$ & 0.06 \\
\hline Globus & $10(71.4 \%)$ & $8(47.1 \%)$ & 0.18 \\
\hline Regurgitation & $12(85.7 \%)$ & $11(64.7 \%)$ & 0.20 \\
\hline Heartburn & $10(71.4 \%)$ & $6(35.3 \%)$ & 0.05 \\
\hline Baseline RSI score & $31.2(8.9)$ & $18.3(9.0)$ & $<0.01$ \\
\hline Baseline GerdQ score & $9.3(3.7)$ & $7.9(3.2)$ & 0.27 \\
\hline \multicolumn{4}{|l|}{ Ambulatory reflux monitoring off PPI } \\
\hline Total acid exposure time (\%) & $3.4(2.7)$ & $3.0(2.4)$ & 0.69 \\
\hline Acid exposure time $>4.0 \%$ & $6(42.9 \%)$ & $5(29.4 \%)$ & 0.51 \\
\hline Total number of reflux events & $73.5(84.9)$ & $76.6(59.6)$ & 0.91 \\
\hline Symptom index & $33.6(34.7)$ & $40.3(33.5)$ & 0.60 \\
\hline Symptom association probability & $95.0(4.2)$ & $88.9(25.7)$ & 0.36 \\
\hline LPR acidic to acidic events & $2.7(4.6)$ & $2.5(3.7)$ & 0.96 \\
\hline LPR acidic to non-acidic events & $2.7(1.5)$ & $1.2(1.9)$ & 0.32 \\
\hline Total LPR events & $5.7(6.4)$ & $5.2(5.3)$ & 0.93 \\
\hline \multicolumn{4}{|l|}{ High-resolution impedance manometry } \\
\hline EGJ baseline pressure (mmHg) & $33.1(18.0)$ & $35.3(31.5)$ & 0.89 \\
\hline Missing & $9(64.3 \%)$ & $12(70.6 \%)$ & \\
\hline Hiatal hernia $(>1 \mathrm{~cm})$ & $6(42.9 \%)$ & $3(17.6 \%)$ & 0.13 \\
\hline CD-LES separation $(\mathrm{cm})$ & $1.0(1.2)$ & $0.3(0.6)$ & 0.07 \\
\hline Median LES IRP (mmHg) & $11.1(5.6)$ & $16.1(6.8)$ & 0.05 \\
\hline Percent of failed swallows & $9.1(13.8)$ & $2.1(5.8)$ & 0.14 \\
\hline$\%$ of bolus clearance complete & $86.4(29.4)$ & $71.9(32.5)$ & 0.26 \\
\hline UES resting pressure $(\mathrm{mmHg})$ & $21.9(23.8)$ & $16.5(26.4)$ & 0.60 \\
\hline UES residual pressure $(\mathrm{mmHg})$ & $2.1(13.3)$ & $4.9(9.3)$ & 0.71 \\
\hline UES length $(\mathrm{cm})$ & $2.9(0.9)$ & $3.2(0.9)$ & 0.56 \\
\hline Baseline salivary pepsin concentration (ng/ml) & $34.6(41.7)$ & 145 (136.4) & 0.01 \\
\hline
\end{tabular}

Continuous data presented as mean (SD) and categorical data as $n(\%)$

$P P I$ proton pump inhibitor, $R S I$ reflux symptom index, $L P R$ laryngopharyngeal reflux, $E G J$ esophago-gastric junction, LES lower esophageal sphincter, IRP integrated relaxation pressure, UES upper esophageal sphincter
Responders to Device + PPI had a significantly lower BMI $\left(24.2 \mathrm{~kg} / \mathrm{m}^{2}\right.$ (4.1) vs $\left.28.6(5.3) ; p=0.02\right)$, a significantly higher salivary pepsin concentration at baseline $(145.0 \mathrm{ng} /$ $\mathrm{ml}(136.4)$ vs $34.6 \mathrm{ng} / \mathrm{ml}$ (41.7); $p=0.01$ ), and tended to have smaller separation between crural diaphragm and lower esophageal sphincter on manometry. Responders to Device + PPI reported a significantly lower baseline RSI score $(18.3$ (9.0) vs $31.2(8.9) ; p<0.01)$ (Table 2$)$.

We also assessed the nine individual items that comprise the complete RSI to identify which items most influenced response. There were four items for which the reduction in RSI sub-score was significantly greater among responders compared to non-responders: \#2 throat clearing $(-1.8$ (SD 1.6) vs $-0.2(0.9) ; p<0.01)$, \#3 excess throat mucus $(-1.9(1.6)$ vs $-0.7(1.4) ; p<0.01)$, \#7 troublesome or annoying cough $(-1.6(1.4)$ vs -0.9 (1.4); $p<0.01)$, and \#9 heartburn, chest pain, indigestion, or stomach acid coming up (-1.3 (1.3) vs $-0.4(0.9)$; $p<0.01)$. 


\section{Secondary Analysis of all Participants that Completed Phase 1 (PPI Alone)}

In the analysis of the 36 participants that completed Phase 1 following PPI therapy, symptom response was met in 13/36 $(36 \%)$. Baseline characteristics between the 13 responders to PPI alone were compared to the 23 non-responders to PPI alone. PPI non-responders had significantly greater number of acidic to non-acidic reflux events and a significantly greater proportion reporting cough. PPI non-responders also tended to have larger hiatal hernia size and higher BMI (Supplemental Table 1).

\section{Safety and Tolerability}

Participants reported a total of 1 mild adverse event related to the device (collar bone discomfort) which resolved without sequelae. There were no serious adverse events. Twentyone participants participated in the 3-month follow-up call. Fourteen $(67 \%)$ reported continued use of the external UES compression device. The seven that discontinued external UES compression device use cited the following reasons: intolerance $(4,57 \%)$, lack of symptom control $(2,29 \%)$, rash $(1,14 \%)$. Sixteen (76\%) reported continued use of their prescribed PPI, whereas five discontinued use due to lack of symptom control.

\section{Discussion}

Effective and safe treatment options are critically needed for patients with LPR. In this two-phase clinical trial, adjunctive use of the external UES compression device (Reflux Band) with PPI compared to PPI alone in suspected LPR was associated with greater symptom relief. Overall $94 \%$ of participants reported reduction in symptom burden after use of the external UES compression device, with 55\% meeting the pre-specified endpoint of symptom response. Participants with symptom response had a lower BMI and tended to have a smaller hiatal hernia size. Further, salivary pepsin concentration at baseline was higher among participants with a symptom response. This first-of-its-kind trial highlights the potential clinical efficacy of the external UES compression device, a well-tolerated noninvasive device that augments UES pressure to reduce esophago-pharyngeal reflux burden, in conjunction with acid suppression for patients with LPR.

PPI therapy is the first-line pharmacologic therapy in GERD, with very high efficacy in healing and maintaining healing of erosive complications of reflux disease in the esophagus. However, the efficacy of PPI therapy is lower for patients with esophageal symptoms of GERD, and wanes even further for patients with extra-esophageal symptoms of GERD such as LPR [28]. One explanation for suboptimal symptom response to acid suppression relates to the well-established fact that symptoms of GERD are not all related to acid [20]. Regurgitation is a symptom perceived due to the mechanical flow of refluxate, regardless of acidity. Further, throat clearing, chronic coughing, and altering vocal cord function are behavioral responses that can be observed in response to several different stimuli, one of which is repeated refluxate exposure [1]. A prior study using a dual hypopharyngeal and esophageal $\mathrm{pH}$ catheter combined with impedance demonstrated that $98 \%$ of LPR events, whether acidic or non-acidic in the distal esophagus, will be non-acidic when they reach the hypopharynx [29]. Thus, effective treatment of LPR requires confidence that the etiology of the symptoms is indeed LPR as well as therapy beyond acid suppression alone. It is therefore not surprising the endpoint of symptom response in this study was met in approximately one-third of participants following PPI therapy alone, and in 55\% following addition of the external UES compression device.

Recent developments have helped clarify the clinical approach to LPR. Since up to $60 \%$ of patients suspected to have LPR are ultimately found to have another non-reflux source of laryngeal symptoms, current guidelines recommend up-front objective testing to understand whether a patient with isolated laryngeal symptoms has an excessive reflux burden $[2,30]$. For patients with laryngeal symptoms and established GERD, trials of PPI therapy are reasonable. Our study suggests that the external UES compression device should be considered for patients with LPR and inadequate symptom relief following PPI therapy. For all patients experiencing extra-esophageal symptoms, consideration for non-GI sources should be explored such as sino-pulmonary conditions, environmental reactions, and laryngeal pathologies. Further, chronic LPR can lead to behavioral changes as manifested by chronic throat clearing, secondary muscle tension dysphonia, and cough. This subset of patients can benefit from a multidisciplinary care model [31].

Our study also highlights that certain phenotypes of LPR, such as those with hiatal hernia, central obesity, and mechanical reflux with cough, are less likely to derive symptom relief with PPI therapy and/or the external UES compression device. Similar to concepts in typical GERD, patients with a large hiatal hernia or elevated intra-abdominal pressure related to central obesity may have excessive reflux burden requiring escalation of management to restore the mechanical dysfunction [32]. Further, in our study, non-responders reported a higher symptom burden at baseline. While this may reflect a higher burden of gastro-esophago-pharyngeal reflux, an intriguing possible explanation is enhanced nociception and laryngeal sensitivity among the non-responder population that is not be responsive to anti-reflux therapies [2] (Fig. 4). This study also highlights the potential of salivary pepsin as a biomarker for LPR. In our study patients 


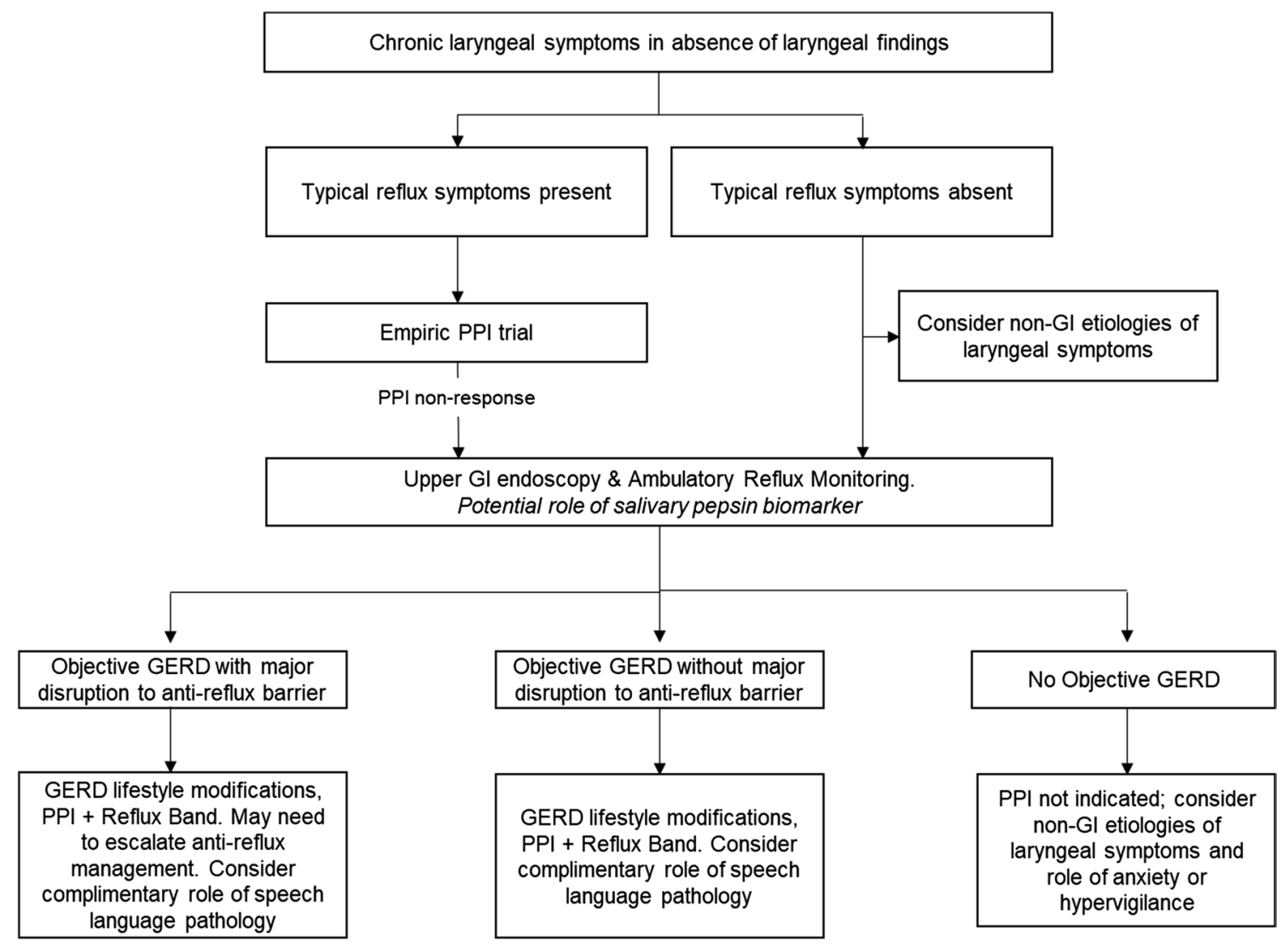

Fig. 4 Personalized clinical approach for suspected LPR. This approach synthesizes results from this study with current practice guidelines to provide a personalized clinical approach for patients

with symptom response to PPI and the external UES compression device had a significantly higher concentration of salivary pepsin at baseline compared to those without symptom response. It is possible that salivary pepsin provides a surrogate measure of proximal refluxate beyond reliance on $\mathrm{pH}$ alone with ambulatory reflux monitoring [27, 33, 34].

Given paucity of previous clinical trial data surrounding the external UES compression device, this study was designed as a single-arm proof-of-concept trial where participants served as their own controls with the intention to assess the device in a randomized sham-controlled trial design if results from this current study were positive. As a result, we recognized inherent limitations of the study design including bias and potential for placebo response. An inherent limitation to all studies related to LPR is the reliance on symptoms as a primary outcome given lack of a well-vetted physiologic gold standard. Hence, we utilized the best available validated patient-reported instrument for LPR, the RSI score, recognizing the potential bias from subjective reporting. While not a large sample population, this sample population is the largest to date for this study type and provides valuable efficacy data for with chronic laryngeal symptoms based on symptom presentation and results of esophageal physiologic testing

future study designs and sample size calculations. Enrollment was significantly challenging due to the ongoing COVID-19 pandemic. While the smaller sample size limited assessment of predictors of response to Device + PPI intervention, the positive results from this prospective trial laid the foundation for a randomized sham-controlled trial that our team is starting to address persisting gaps in the field. Finally, there is potential of cross-over of PPI effect during Phase 2 of the study, however, given that the literature demonstrates healing of esophagitis within 2 weeks of PPI use it was determined that 4 weeks of PPI in Phase 1 was sufficient and would strengthen feasibility.

In conclusion, the external UES compression device (Reflux Band) is a potentially efficacious noninvasive therapy for LPR. This study highlights its complimentary role with PPI therapy in reducing symptom burden in patients with LPR. Further, particular factors may increase likelihood of treatment response, such as higher baseline salivary pepsin concentration, lower BMI, and smaller defect at the anti-reflux barrier, highlighting the need for personalized approaches to LPR. 
Supplementary Information The online version contains supplementary material available at https://doi.org/10.1007/s10620-021-07172-2.

Author's contribution RY, SG, and SW oversaw the study; RY, MG, $\mathrm{JC}, \mathrm{SW}$, and MC acquired data; RY, SW, MG, JC, and AK analyzed and interpreted data; RY, JEP, SG, AK, SW, MG, JC, PMK, DF, MC, AMVH, MC, and PW drafted the manuscript; RY, JEP, SG, SW, AK, MG, PMK, DF, MC, AMVH, MC, PW, and JC critically revised the manuscript for important intellectual content; RY, JEP, SG, SW, MG, AK, JC, PMK, DF, MC, AMVH, MC, and PW finalized the manuscript.

Funding This study was supported by the ACG Junior Faculty Development Award (PI Yadlapati).

Data availability De-identified individual participant data that underlie the reported results will be made available 3 months after publication for a period of 5 years after the publication date. Proposals for access should be sent to corresponding author. The study protocol is included as a data supplement available with the online version of this article.

\section{Declarations}

Conflict of interest RY: Consultant for Medtronic (Institutional), Ironwood Pharmaceuticals (Institutional), Phathom Pharmaceuticals. Medical Advisory board with Stock Options: RJS Mediagnostix. Research funding: Ironwood Pharmaceuticals. SW: Consultant for Boston Scientific, Medtronic, Cernostics, Exact Sciences and Interpace. Supported by the NIH/NIDDK (U34 DK124174) and University of Colorado Department of Medicine Outstanding Early Scholars Program. None: JC, MC, MC, DF, MG, SG, AK, PMK, AMVH, JEP, and PW.

\section{References}

1. Cohen SM, Pitman MJ, Noordzij JP et al. Management of dysphonic patients by otolaryngologists. Otolaryngol Head Neck Surg 2012;147:289-294.

2. de Bortoli N, Nacci A, Savarino E et al. How many cases of laryngopharyngeal reflux suspected by laryngoscopy are gastroesophageal reflux disease-related? World J Gastroenterol 2012;18:4363-4370.

3. Koufman JA, Amin MR, Panetti M. Prevalence of reflux in 113 consecutive patients with laryngeal and voice disorders. Otolaryngol Head Neck Surg 2000;123:385-388.

4. Ruiz R, Jeswani S, Andrews K et al. Hoarseness and laryngopharyngeal reflux: a survey of primary care physician practice patterns. JAMA Otolaryngol Head Neck Surg 2014;140:192-196.

5. Koufman JA. The otolaryngologic manifestations of gastroesophageal reflux disease (GERD): a clinical investigation of 225 patients using ambulatory 24-hour $\mathrm{pH}$ monitoring and an experimental investigation of the role of acid and pepsin in the development of laryngeal injury. Laryngoscope 1991;101:1-78.

6. Olson NR. Laryngopharyngeal manifestations of gastroesophageal reflux disease. Otolaryngol Clin North Am 1991;24:1201-1213.

7. Vakil N, van Zanten SV, Kahrilas P et al. The Montreal definition and classification of gastroesophageal reflux disease: a global evidence-based consensus. Am J Gastroenterol 2006;101:1900-1920 (quiz 1943)

8. Yadlapati R, Katzka DA. Laryngopharyngeal reflux is an eternally rolling boulder. Clin Gastroenterol Hepatol 2020;18:1431-1432.

9. Carrau RL, Khidr A, Crawley JA et al. The impact of laryngopharyngeal reflux on patient-reported quality of life. Laryngoscope 2004;114:670-674.
10. Carrau RL, Khidr A, Gold KF et al. Validation of a quality-of-life instrument for laryngopharyngeal reflux. Arch Otolaryngol Head Neck Surg 2005;131:315-320.

11. Francis DO, Rymer JA, Slaughter JC et al. High economic burden of caring for patients with suspected extraesophageal reflux. Am J Gastroenterol 2013;108:905-911.

12. Lenderking WR, Hillson E, Crawley JA et al. The clinical characteristics and impact of laryngopharyngeal reflux disease on healthrelated quality of life. Value Health 2003;6:560-565.

13. Lechien JR, Dapri G, Dequanter D et al. Surgical treatment for laryngopharyngeal reflux disease: a systematic review. JAMA Otolaryngol Head Neck Surg 2019;145:655-666.

14. Lechien JR, Saussez S, Schindler A et al. Clinical outcomes of laryngopharyngeal reflux treatment: a systematic review and metaanalysis. Laryngoscope 2019;129:1174-1187.

15. Karkos PD, Wilson JA. Empiric treatment of laryngopharyngeal reflux with proton pump inhibitors: a systematic review. Laryngoscope 2006;116:144-148.

16. Qadeer MA, Phillips CO, Lopez AR et al. Proton pump inhibitor therapy for suspected GERD-related chronic laryngitis: a metaanalysis of randomized controlled trials. Am J Gastroenterol 2006;101:2646-2654.

17. Krill JT, Naik RD, Higginbotham T et al. Association between response to acid-suppression therapy and efficacy of antireflux surgery in patients with extraesophageal reflux. Clin Gastroenterol Hepatol 2017;15:675-681.

18. So JB, Zeitels SM, Rattner DW. Outcomes of atypical symptoms attributed to gastroesophageal reflux treated by laparoscopic fundoplication. Surgery 1998;124:28-32.

19. Swoger J, Ponsky J, Hicks DM et al. Surgical fundoplication in laryngopharyngeal reflux unresponsive to aggressive acid suppression: a controlled study. Clin Gastroenterol Hepatol 2006;4:433-441.

20. Sharma P, Yadlapati R. Pathophysiology and treatment options for gastroesophageal reflux disease: looking beyond acid. Ann $N$ Y Acad Sci 2020;1486:3-14.

21. Patel D, Vaezi MF. Normal esophageal physiology and laryngopharyngeal reflux. Otolaryngol Clin North Am 2013;46:1023-1041.

22. Babaei A, Venu M, Naini SR et al. Impaired upper esophageal sphincter reflexes in patients with supraesophageal reflux disease. Gastroenterology 2015;149:1381-1391.

23. Shaker R, Babaei A, Naini SR. Prevention of esophagopharyngeal reflux by augmenting the upper esophageal sphincter pressure barrier. Laryngoscope 2014;124:2268-2274.

24. Silvers S, Vaezi M, Vakil N et al. Prospective study of upper esophageal sphincter assist device for treating extraesophageal reflux. Otolaryngology Open J 2016;2:31-38.

25. Belafsky PC, Postma GN, Koufman JA. Validity and reliability of the reflux symptom index (RSI). $J$ Voice 2002;16:274-277.

26. Dent J, Vakil N, Jones R et al. Accuracy of the diagnosis of GORD by questionnaire, physicians and a trial of proton pump inhibitor treatment: the Diamond Study. Gut 2010;59:714-721.

27. Yadlapati R, Kaizer A, Greytak M, et al. Diagnostic performance of salivary pepsin for gastroesophageal reflux disease. Dis Esophagus 2020.

28. Boeckxstaens G, El-Serag HB, Smout AJ et al. Symptomatic reflux disease: the present, the past and the future. Gut 2014;63:1185-1193.

29. Yadlapati R, Carroll TL, Fenn J et al. Combined hypopharyngealesophageal impedance-Ph monitoring: a pilot study on physiology of laryngopharyngeal reflux and normative values. Gastroenterology 2020;158:S-1059-S-1060.

30. Gyawali CP, Carlson DA, Chen JW et al. ACG clinical guidelines: clinical use of esophageal physiologic testing. Am J Gastroenterol 2020;115:1412-1428. 
31. Schneider SL, Clary MS, Fink DS et al. Voice therapy associated with a decrease in the reflux symptoms index in patients with voice complaints. Laryngoscope 2019;129:1169-1173.

32. Yadlapati R, Pandolfino JE. Personalized approach in the work-up and management of gastroesophageal reflux disease. Gastrointest Endosc Clin N Am 2020;30:227-238.

33. Zhang M, Pandolfino JE, Zhou X et al. Assessing different diagnostic tests for gastroesophageal reflux disease: a systematic review and network meta-analysis. Therap Adv Gastroenterol 2019;12:1756284819890537.
34. Yadlapati R, Craft J, Adkins CJ et al. The upper esophageal sphincter assist device is associated with symptom response in reflux-associated laryngeal symptoms. Clin Gastroenterol Hepatol 2018;16:1670-1672.

Publisher's Note Springer Nature remains neutral with regard to jurisdictional claims in published maps and institutional affiliations. 